\title{
Preterm Fetal Hypoxia-Ischemia Causes Hypertonia and Motor Deficits in the Neonatal Rabbit: A Model for Human Cerebral Palsy?
}

\author{
Matthew Derrick, ${ }^{1}$ Ning Ling Luo, ${ }^{3}$ Joanne C. Bregman, ${ }^{1}$ Tamas Jilling, ${ }^{1}$ Xinhai Ji, ${ }^{1}$ Kara Fisher, ${ }^{1}$ Candece L. Gladson, ${ }^{2}$ \\ Douglas J. Beardsley, ${ }^{3}$ Geoffrey Murdoch, ${ }^{4}$ Stephen A. Back, ${ }^{3,5 *}$ and Sidhartha Tan ${ }^{1 \star}$ \\ ${ }^{1}$ Department of Pediatrics, Northwestern University, and Evanston Northwestern Healthcare, Evanston, Illinois 60201, 2Department of Neuro-Pathology, \\ University of Alabama at Birmingham, Birmingham, Alabama 35243, and Departments of ${ }^{3}$ Pediatrics, ${ }^{4}$ Pathology, and ${ }^{5}$ Neurology, Oregon Health Sciences \\ University, Portland, Oregon 97201
}

Prenatal hypoxia-ischemia to the developing brain has been strongly implicated in the subsequent development of the hypertonic motor deficits of cerebral palsy (CP) in premature and full-term infants who present with neonatal encephalopathy. Despite the enormous impact of $\mathrm{CP}$, there is no animal model that reproduces the hypertonia and motor disturbances of this disorder. We report a rabbit model of in utero placental insufficiency, in which hypertonia is accompanied by marked abnormalities in motor control. Preterm fetuses (67-70\% gestation) were subjected to sustained global hypoxia. The dams survived and gave spontaneous birth. At postnatal day 1, the pups that survived were subjected to a battery of neurobehavioral tests developed specifically for these animals, and the tests were videotaped and scored in a masked manner. Newborn pups of hypoxic groups displayed significant impairment in multiple tests of spontaneous locomotion, reflex motor activity, and the coordination of suck and swallow. Increased tone of the limbs at rest and with active flexion and extension were observed in the survivors of the preterm insult.

Histopathological studies identified a distinct pattern of acute injury to subcortical motor pathways that involved the basal ganglia and thalamus. Persistent injury to the caudate putamen and thalamus at P1 was significantly correlated with hypertonic motor deficits in the hypoxic group. Antenatal hypoxia-ischemia at preterm gestation results in hypertonia and abnormalities in motor control. These findings provide a unique behavioral model to define mechanisms and sequelae of perinatal brain injury from antenatal hypoxia-ischemia.

Key words: abruptio placentae; asphyxia; behavior; development; dystonia; cerebral white matter; locomotion; motor control; neurologic examination; prematurity; spasticity

\section{Introduction}

Hypoxic-ischemic brain injury results in cerebral palsy (CP), mental retardation, or learning disabilities in surviving children (Robertson and Finer, 1985). It is now widely recognized that the majority of cases of term neonatal encephalopathy are related to hypoxic-ischemic brain injury that occurs in utero from a variety of intrapartum conditions (Badawi et al., 1998). Hypoxia-ischemia $(\mathrm{H}-\mathrm{I})$ is also associated with subsequent cerebral injury in a disproportionately high percentage of the survivors of premature birth (Volpe, 2001). The incidence of CP increases dramatically with the decline in gestational age at birth, such that $4-12 \%$ of

Received June 4, 2003; revised 0ct. 20, 2003; accepted 0ct. 20, 2003.

This work was supported by National Institutes of Health Grants NS41343 and NS41476 to S.A.B. and NS43285 to S.T., and March of Dimes Birth Defects Foundation 294 to S.T. We acknowledge Elizabeth Brady and Jie He for technical support. We are grateful to Dr. Barry Russman for his helpful advice.

*S.A.B. and S.T. contributed equally to this work.

Correspondence should be addressed to Dr. Sidhartha Tan, Department of Pediatrics, Northwestern University, 2650 Ridge Avenue, Evanston, IL 60201. E-mail: sidtan@northwestern.edu.

DOI:10.1523/JNEUROSCI.2816-03.2004

Copyright $\odot 2004$ Society for Neuroscience $\quad$ 0270-6474/04/240024-11\$15.00/0 infants with a birth weight $<1000$ gm are affected (Schmidt et al., 2001).

In view of the major contribution of intrapartum risk factors and prematurity to subsequent neurological morbidity and mortality, studies are needed that address the underlying mechanisms of brain injury that occur in utero to the immature and near-term fetal CNS. The lack of a suitable fetal animal model that reproduces the motor deficits of $\mathrm{CP}$ has hampered definition of these mechanisms. Most previous studies have employed models of cerebral hypoxia-ischemia in postnatal animals. Typically, a significant mortality was observed or the animals recovered completely and neurobehavioral deficits were difficult to elicit (Wright and Rang, 1990).

We have developed a model of global hypoxia-ischemia in the near-term fetal rabbit subjected to uterine ischemia that accurately reflects acute placental insufficiency in humans (Tan et al., 1999;Derrick et al., 2001). Both sustained and intermittent fetal hypoxia-ischemia resulted in marked increases in the production of reactive nitrogen and oxygen species in the fetal brain, and antioxidant therapy reduced the degree of brain injury (Tan et al., 1998; Tan et al., 1999; Tan et al., 2001). In vitro culture of neural 
cells dissociated from the ischemic fetal brains demonstrated increases in both early necrotic cell death and delayed apoptosis (Derrick et al., 2001).

In the present study, a new neurobehavioral battery of tests was used to characterize the motor performance of newborn pups after sustained hypoxia-ischemia to the preterm rabbit fetus at $67-70 \%$ gestation. Fetuses were allowed to deliver spontaneously at term gestation and then subjected to the neurobehavioral battery before histological analysis of CNS injury. This model offers the distinct advantages that, similar to the human newborn, the newborn rabbit has immature locomotor function and the cerebral hemispheres are not fully myelinated. Uterine ischemia in the dams resulted in a distinct neurobehavioral phenotype in the newborn pups, which was characterized by an increase in forelimb tone that was significantly correlated with histological evidence of persistent injury to subcortical motor pathways involving the basal ganglia and anterior thalamus. These studies provide the first animal model in which antecedent insult during development of the immature CNS results in a constellation of hypertonic motor deficits at term birth that are consistent with those observed in $\mathrm{CP}$.

\section{Materials and Methods}

Animal surgery. In vivo global hypoxia-ischemia of fetuses was induced by uterine ischemia in timed pregnant New Zealand white rabbits (Myrtle's Rabbits, Thompson Station, Tennessee) as described previously (Tan et al., 1998; Derrick et al., 2001). Accurate timing of gestation was ensured by observing the mating of the rabbits and limiting the exposure of does to male rabbits to $5 \mathrm{~min}$ by the vendor. Briefly, the dams were anesthetized with intravenous fentanyl $(75 \mu \mathrm{g} / \mathrm{kg} / \mathrm{hr})$ and droperidol $(3.75 \mathrm{mg} / \mathrm{kg} / \mathrm{hr})$, and bag and mask ventilation was provided to maintain normal arterial $\mathrm{pH}$ (7.35-7.45), $\mathrm{PcO}_{2}$ (32-45 torr), and $\mathrm{Po}_{2}$ (70-100 torr). Thereafter, the dams underwent spinal anesthesia by the administration of $0.75 \%$ bupivicaine through a $25 \mathrm{G}$ spinal needle placed at L4-L5 intervertebral space. The fentanyl and droperidol dose was reduced by $60 \%$ to allow the dam to breathe spontaneously through a mask. Uterine ischemia, which resulted in fetal hypoxia, was induced with a $4 \mathrm{~F}$ Fogarty arterial embolectomy catheter (Baxter Health Care Corporation, Santa Ana, CA). The catheter was introduced into the left femoral artery, advanced $10 \mathrm{~cm}$ into the descending aorta to above the uterine and below the renal arteries, and the balloon was inflated with 300 $\mu \mathrm{l}$ of saline. Right lower extremity blood pressure was monitored by Doppler (Mini Dopplex D500; Huntleigh Technology, Luton, UK) to ensure continued ischemia. At the end of the procedure, the balloon was deflated and the catheter was removed. The femoral artery was repaired with 7-0 sutures, and the skin was closed with $3-0$ sutures. The mother was returned to her cage. The dams either underwent hysterotomy $24 \mathrm{hr}$ later at $23 \mathrm{~d}$ gestation or were allowed to deliver in a nest box at term $(31.5$ d). All pups found dead in the newborn period were concluded to be perinatal stillbirths, because their weights were similar to that of their live littermates.

Hypoxia-ischemia protocol. Preterm animals at 21-22 d gestation [67$70 \%$ gestation; embryonic day (E) 21-E22] underwent sustained uterine ischemia for $30,37,38$, or $40 \mathrm{~min}$. This was done in an attempt to model an insult of placental insufficiency to premature infants. This protocol in near-term rabbits results in global hypoxia to the fetus that is accompanied by immediate fetal bradycardia (from 180 to $80 \mathrm{bpm}$ ) and an immediate drop in microvascular blood flow to the cerebral cortex, as determined by Laser Doppler measurements (Perimed, Jarfalla, Sweden). The numbers of dams and pups in each group are shown in Table 1.

Behavioral testing. After natural delivery, on postnatal day (P) 1 , four pups per litter were selected at random for behavioral testing. Thus, behavioral testing was done $10 \mathrm{~d}$ after the hypoxic insult at E22 or $11 \mathrm{~d}$ after the insult at E21. If the litter size was less than four live pups, all live pups were evaluated. This protocol was selected to minimize the effect of specific litters on overall group effect. The design of the behavioral battery was based on the capabilities of the newborn pup at P1. For example,
Table 1. Number of dams and pups in each group

\begin{tabular}{llll}
\hline Group & $\begin{array}{l}\text { Number of } \\
\text { dams }\end{array}$ & $\begin{array}{l}\text { Number of } \\
\text { pups }\end{array}$ & $\begin{array}{l}\text { Number of pups undergoing } \\
\text { behavioral testing }\end{array}$ \\
\hline Control & 6 & 58 & 24 \\
I & 3 & 28 & 12 \\
II (E22, 30 min) & 3 & 26 & 12 \\
II (E21 $\geq 37)$ & 6 & 51 & 22 \\
II (E22 $\geq 37)$ & 6 & 55 & 23 \\
\hline
\end{tabular}

Table 2. Physiological variables

\begin{tabular}{lcc}
\hline & Control & Hypoxia (E22, 37-40 min) \\
\hline Litter size & $9.2 \pm 0.6$ & $8.6 \pm 1.1$ \\
Weight of dead pups & $46 \pm 6$ & $45 \pm 2$ \\
Weight of live pups & $58.3 \pm 0.9$ & $54.8 \pm 1.4^{*}$ \\
Weight of hypertonic pups & & $51 \pm 1$ \\
Weight of nonhypertonic pups & $58 \pm 1$ & $59 \pm 3$ \\
Rectal temperature $\left({ }^{\circ} \mathrm{C}\right)$ & $36.4 \pm 0.2$ & $35.9 \pm 0.2$ \\
Heart rate & $214 \pm 9$ & $214 \pm 14$ \\
Mixed venous blood & & \\
$\mathrm{pH}$ & $7.48 \pm 0.04$ & $7.44 \pm 0.05$ \\
$\mathrm{Pco}_{2}$ & $26.7 \pm 3.0$ & $29.9 \pm 6.6$ \\
$\mathrm{Po}{ }_{2}$ & $39.1 \pm 9.0$ & $30.7 \pm 4.7$ \\
$\quad$ Base excess & $-7.8 \pm 1.7$ & $-8.6 \pm 0.7$ \\
Sodium (mM) & $148 \pm 5$ & $138 \pm 1$ \\
Potassium (mM) & $6.4 \pm 0.9$ & $5.3 \pm 0.7$ \\
Calcium ionized (mm) & $0.98 \pm 0.09$ & $1.25 \pm 0.09$ \\
$\quad$ Glucose (mg/dl) & $125 \pm 11$ & $121 \pm 12$ \\
\hline
\end{tabular}

Values expressed as mean \pm SEM; no significant difference between the groups except when marked ${ }^{*} p<0.05$; $t$ test).

at P1, the pups displayed spontaneous locomotion and responded behaviorally to tactile and noxious stimuli but not to light or sound. The pups were evaluated in their nest box. Rectal temperatures were recorded at the time of behavior testing in a subset of animals, and there were no significant differences between control and $40 \mathrm{~min}$ uterine ischemia groups (Table 2).

For each animal, the testing was videotaped and scored on a scale of $0-3$ ( 0 , worst; 3 , best) by two blinded observers. A description of the scoring system is given in Table 3. A videotape and movie of the results is also provided (available at www.jneurosci.org). Locomotion on a flat surface was assessed by grading the amount of spontaneous movement of the head, trunk, and limbs. Tone was assessed by active flexion and extension of the forelimbs and hindlimbs and scored $(0-4)$ according to the Ashworth scale (Damiano et al., 2002). The righting reflex was assessed when the pups were placed on their backs. Suck and swallow were assessed by introduction of formula (Similac with iron; Abbott Labs, Abbott Park, IL) into the pup's mouth with a plastic pipette.

To determine whether the preterm animals displayed adverse physiological consequences that might interfere with neurobehavioral testing, heart rate was measured in a subgroup of randomly selected pups by 8 $\mathrm{MHz}$ Doppler transducer (Mini Dopplex D500; Huntleigh Technology) at P1. Thereafter, the pups were killed, and mixed venous and arterial blood samples were collected. Arterial blood gas determinations were made along with sodium, potassium, and ionized calcium on an ABL 700 Series Analyzer (Radiometer Medical, Copenhagen, Denmark). Serum glucose was determined on a SureStepFlexx Meter (Lifescan, Milpitas, CA).

Histological studies. After behavioral testing, pups at P1 were anesthetized by a single intramuscular dose of a combination of ketamine (35-40 mg/kg), acepromazine (0.75-1.0 mg/kg), and xylazine (3-5 mg/ $\mathrm{kg}$ ) and then killed by decapitation. The brains from five control animals and nine experimental animals were immersion fixed for $24 \mathrm{hr}$ at $4^{\circ} \mathrm{C}$ in $4 \%$ paraformaldehyde in $0.1 \mathrm{~m}$ phosphate buffer, $\mathrm{pH}$ 7.4. The brains were systematically cut into $2 \mathrm{~mm}$ thick coronal blocks with a matrix tissue slicer to uniformly sample similar regions in each brain. Alternate $2 \mathrm{~mm}$ blocks of tissue were embedded in paraffin and sectioned at $8 \mu \mathrm{m}$ or 
Table 3. Neurobehavioral scoring protocol for rabbit pups at $\mathrm{P} 1$

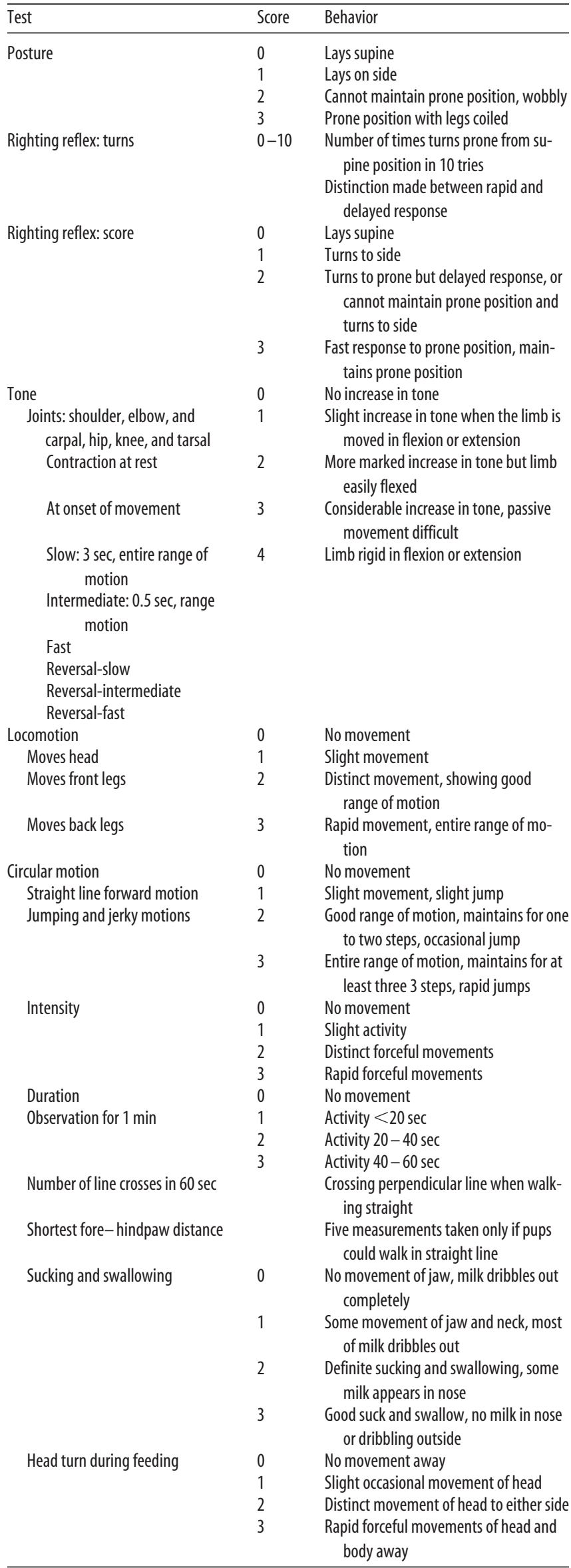

sectioned free-floating at $50 \mu \mathrm{m}$ with a vibrating microtome. The regions sampled for paraffin embedding contained from rostral to caudal the midseptal nuclei of the forebrain, the ventral posterolateral nucleus of the thalamus and overlying hippocampal formation, the facial motor nucleus of the pons and overlying cerebellum, and the cervico-medullary junction. Free-floating sections were cut from a $2 \mathrm{~mm}$ block of tissue, which contained the head of the caudate nucleus and the anterior thalamic nuclei. These sections were stained with the biotinylated microglia-macrophage marker Bandeiria griffonia isolectin B4 (1:100; L2140; Sigma, St. Louis, MO) and were visualized with rhodamine red X-conjugated streptavidin (1:400; 016-290-084; Jackson ImmunoResearch, West Grove, PA).

Paraffin-embedded tissue sections were immunohistochemically stained in duplicate for the macrophage and activated microglial marker HAM 56. Sections were first processed for antigen retrieval by immersion in $50 \mathrm{~mm}$ Tris- $\mathrm{HCl}, \mathrm{pH} \mathrm{10}$, for $25 \mathrm{~min}$ and in a bath that was steam heated to $100^{\circ} \mathrm{C}$ in a rice cooker. Sections were cooled in room air for $20 \mathrm{~min}$ in this solution and then processed for immunoperoxidase histochemistry as follows. Sections were treated with $0.3 \%$ hydrogen peroxidase for 5 min, washed in $50 \mathrm{~mm}$ Tris- $\mathrm{HCl}, \mathrm{pH} 7.4$, and reacted overnight at $2-4^{\circ} \mathrm{C}$ with the HAM 56 IgM primary antibody (MO632; Dako, Carpinteria, CA) diluted 1:200 in Tris buffer containing 1\% BSA (Sigma). Thereafter, sections were incubated with a biotinylated anti-mouse IgM $\mu$ chainspecific secondary antibody (1:100; BA2020; Vector Laboratories, Burlingame, CA) using a Vectastain Elite ABC peroxidase kit (PK6100; Vector Laboratories) and following the instructions of the manufacturer.

The density and total area of HAM 56-labeled microglia were determined in a masked manner under bright-field with a Leica (Nussloch, Germany) DMRX upright microscope equipped with an Orca-ERcooled CCD digital camera (C4742-95; Hamamatsu, Shizouka, Japan) that was interfaced to the Open Lab 3.0.4 image analysis software (morphometry package; Improvision, Boston, MA). For each region analyzed, a single digitized image was acquired with a $20 \times$ phase contrast objective (HC PL Fluotar; numerical aperature, 0.50, $\mathrm{pH} 2$; Leica), in which the number of HAM 56-labeled cells appeared to be maximal. All images were acquired with the same exposure settings. The region analyzed was verified by the morphology and distribution of neurons visualized by phase contrast microscopy. For each digitized image, both the number and total area of HAM 56-labeled microglia were determined by a density slice-threshold analysis in which minimal pixel intensity was set at 40 pixel units.

Fluorescence in situ detection of DNA fragmentation $24 \mathrm{hr}$ after insult. Hysterotomy was performed on four dams $24 \mathrm{hr}$ after 40 min uterine ischemia, and the fetuses were delivered. The brains from these E23 fetuses were fixed for $24 \mathrm{hr}$ by immersion in ice-cold $4 \%$ paraformaldehyde in $0.1 \mathrm{M}$ phosphate buffer before storage at $2-4^{\circ} \mathrm{C}$ in PBS. A total of 18 hypoxic fetal brains was compared with six control brains from two litters of E23 control animals. Free-floating $50 \mu \mathrm{m}$ coronal sections of the entire brain were sectioned serially in ice-cold PBS on a Leica VTS-1000 vibrating microtome and analyzed at $250 \mu \mathrm{m}$ intervals. Tissue sections were mounted and air-dried on subbed slides. In preliminary studies, tissue sections were either permeabilized for $5 \mathrm{~min}$ in 1:1 ethanol:acetic acid $\left(-20^{\circ} \mathrm{C}\right)$ for $10 \mathrm{~min}$ in Triton $\mathrm{X}-100$ or for $5 \mathrm{~min}$ at room temperature in proteinase $\mathrm{K}(20 \mu \mathrm{g} / \mathrm{ml}$ in PBS). Treatment with ethanol:acetic acid or Triton X-100 underestimated the extent of terminal deoxynucleotidyl transferase-mediated biotinylated UTP nick end labeling (TUNEL) when compared with the proteinase K treatment, which was used for the analysis of injury. An ApopTag-fluorescein in situ DNA fragmentation detection kit (Intergen, Purchase, NY) was used to visualize TUNEL-labeled nuclei, as reported previously (Back et al., 2001). Tissue sections were counterstained with Hoechst 33324.

Our study was approved by the Animal Review Committee of the Evanston Northwestern Healthcare Research Institute. All animals received humane care in compliance with the Principles of Laboratory Care formulated by the National Society for Medical Research and with the National Institute of Health's Guide for the Care and Use of Laboratory Animals prepared by the National Academy of Sciences.

Statistical analysis. The animals were analyzed in four groups: control nonischemic animals $(n=129)$, hypoxia for $30 \mathrm{~min}$ at E22 $(n=26)$, 

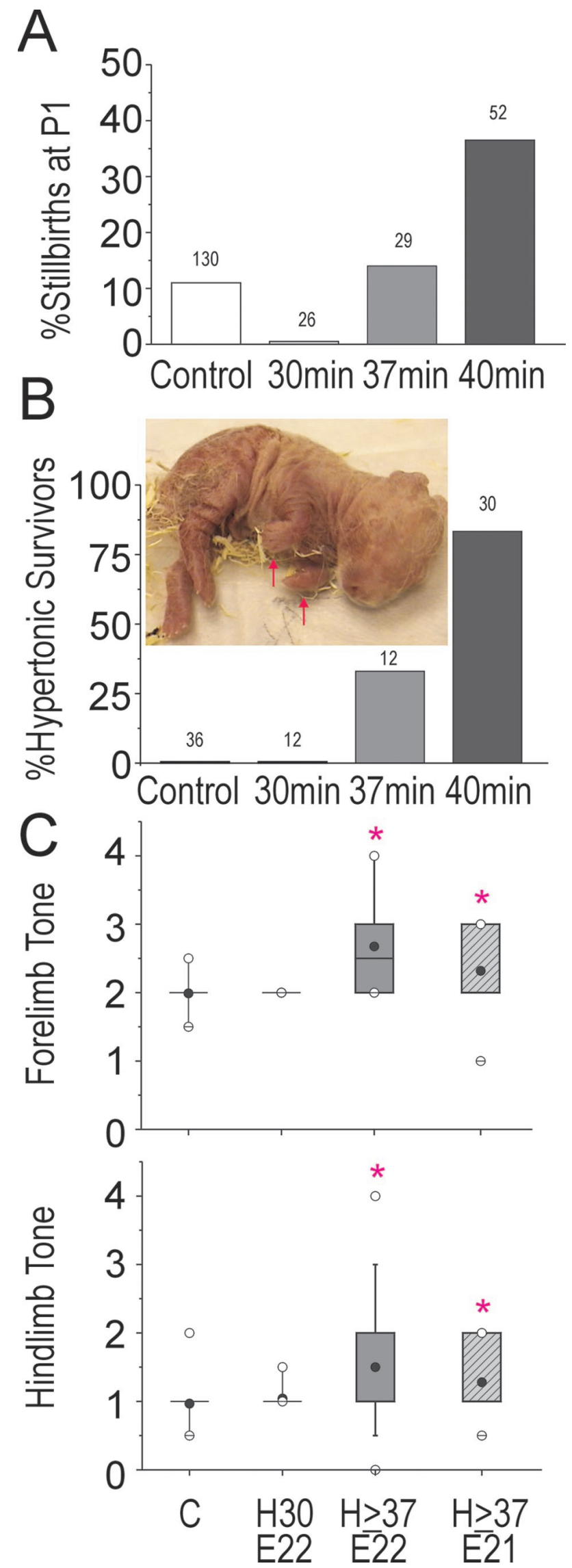

Figure 1. A, Incidence of stillbirths noted at $\mathrm{P} 1$ in control and after sustained hypoxia for 30 , 37, or $40 \mathrm{~min}$ at E22. The number on each bar shows the number of pups in each group. $B$, Hypertonicity rates at P1 of control and after sustained hypoxia for 30, 37, or 40 min at hypoxia for 37-40 $\mathrm{min}$ at E22 $(n=102)$, and hypoxia for 37-40 min at E21 $(n=61)$. The latter two groups were both labeled as $\geq 37 \mathrm{H}-\mathrm{I}$. The ordinal scores in these groups were analyzed by the Kruskal-Wallis test with post hoc Wilcoxon signed rank comparisons with $\alpha$ correction $=$ 0.0224 , in view of the multiple comparisons. Righting reflex and shortest forelimb-hindlimb paw distance was analyzed by ANOVA with Student Newman Keuls post hoc comparison of means. Other variables, including weight, heart rate, blood gases, and electrolytes groups, were compared with a two-sample $t$ test. Microglial number and area were analyzed by Wilcoxon signed rank comparison. The association of activated microglial (cells $/ \mathrm{mm}^{2}$ and area) with hypertonia (Ashworth scale score) was analyzed by Fisher's exact test. All statistical analysis was done using SAS for Windows, Release 8.2.

\section{Results}

We determined the effects of sustained hypoxia of 30-40 min duration on the 22 d gestation (E22) fetus. As expected from human studies of acute placental insufficiency (Saftlas et al., 1991), stillbirths were observed in the hypoxic animals. The incidence of stillbirths increased with increased duration of sustained hypoxia beyond $30 \mathrm{~min}$ (Fig. $1 \mathrm{~A}$ ). The mean weight of the stillborn pups (control group) was $21 \%$ lower than live-born control pups, and a similar decrease of $17 \%$ was observed for the hypoxic group. This suggested that the timing of prenatal death was similar in both groups. We next determined the responses of the newborn survivors to a neurobehavioral battery of tests (Table 3 ). Animals subjected to $30 \mathrm{~min}$ of hypoxia were indistinguishable from controls (Figs. 1-4). However, neurobehavioral changes were observed in animals subjected to $\geq 37 \mathrm{~min}$ of sustained hypoxia. The most striking finding was the presence of overt changes in posture and tone in the $\mathrm{P} 1$ survivors (Fig. $1 \mathrm{~B}$ ). These pups typically had a flexed posture of their limbs at rest (Fig. $1 B$, inset) (supplementary videotape; available at www.jneurosci.org). In the animals subjected to $40 \mathrm{~min}$ of hypoxia, $37 \%$ of the births were stillbirths, and of the survivors, $83 \%$ of the pups were hypertonic. There was a significant increase in the hypertonicity score for the two groups of animals subjected to $\geq 37 \mathrm{~min}$ of sustained hypoxia at E22 and E21 (Fig. 1C).

\section{Sustained hypoxia at preterm gestation results in hypertonic motor deficits at P1}

In a subgroup of pups born after 40 min of hypoxia at E22, we determined the types of abnormalities in forelimb tone (Fig. 2). Tone was evaluated according to the recent recommendations of the National Institutes of Health Workshop on Childhood Motor Disorders (Sanger et al., 2003) and was rated at rest and during slow- or fast-applied stretch to the joint. In general, animals in the hypoxic group displayed increased forelimb tone at rest (Fig. $2 C-F$ ) compared with control animals (Fig. 2A). This finding was commonly accompanied by fixed involuntary postures of the forelimbs at rest (Fig. 2, insets, images of test animals). With slow or fast stretch of the joint in flexion or extension, there was typically no velocity-dependent increase in resistance of the muscles to stretch. A subset of the animals did display a small increase in tone with slow flexion of the limb (Fig. $2 B$ ). The majority of animals displayed a similar degree of resistance of the muscle to

E22. The number on each bar shows the number of pups in each group. Inset shows a pup subjected to 40 min at E22 demonstrating abnormal posture (arrows). C, Hypertonicity score of forelimbs and hindlimbs in control (C) and after sustained hypoxia for 30 min at E22 (H30, E22), 37- 40 min at E22 ( $\mathrm{H} \geq 37, \mathrm{E22})$, or at E21 ( $\mathrm{H} \geq 37, \mathrm{E} 21)$. Data are shown as a box and whisker plot. Black circles, Mean; white circles, 99th percentile; groups significantly different by Kruskal-Wallis test. ${ }^{*} p<0.0224$; Wilcoxon signed rank test compared with controls. 
stretch at slow and fast velocity (Fig. $2 D-F)$ with flexion, extension, or both. Another subset showed a small decrease in tone with active flexion of the limb (Fig. $2 C, D)$.

Sustained hypoxia at preterm gestation results in impaired locomotor activity Because the majority of survivors of prolonged preterm hypoxia displayed tone abnormalities, we determined whether this insult also resulted in impaired locomotor activity. When we compared mild (30 $\mathrm{min})$ and severe $(\geq 37 \mathrm{~min}$ ) hypoxia with controls, we found that the $\geq 37 \mathrm{~min}$ hypoxia groups were significantly more impaired in the quality, intensity, and duration of control of the head and limbs. Figure 3, $A-C$, shows the significantly lower motor activity scores of $\geq 37 \mathrm{~min}$ hypoxia groups that were determined for control of the head (Fig. 3A), forelimbs (Fig. 3B), and hindlimbs (Fig. 3C). These impairments in locomotor control were supported by scores that rated spontaneous forward motor activity (Fig. 3D), the number of straight-line crosses in $60 \mathrm{sec}$ (Fig. $3 E$ ), and circling (Fig. $3 F$ ). Because the forelimbs were generally stronger than the hindlimbs, the more mildly affected pups could move in circles but could not move forward in a single direction. The most severely affected pups were not able to purposefully move the head or limbs. However, in those pups that were able to walk in a straight line, the shortest distance between the forelimb and hindlimb paws was used as a measure of the ability of the hindlimbs to keep up with the forelimbs, and it was not different between the controls and hypoxia groups (data not shown).

Motor reflexes were impaired in the hypoxia groups that sustained $\geq 37 \mathrm{~min}$. The righting reflex was significantly affected in the hypoxia groups (Fig. 4A). The lower scores for the severe hypoxia groups reflected a spectrum of impairment. When placed supine, the mildly affected pups returned to a prone position over a longer time period, whereas more severely affected animals could only turn to the side or remain supine. When we counted the number of successful turns to a prone position in 10 attempts, the hypoxic group, $\geq 37 \mathrm{~min}$ at E22, had significantly fewer turns $(6 \pm 1)$ compared with other groups (controls, $9 \pm 0$; hypoxia, $30 \mathrm{~min}$, $10 \pm 0$; hypoxia, $\geq 37$ min at $\mathrm{E} 21,8 \pm 1$; mean $\pm \mathrm{SEM}$ ).

The coordination of suck and swallow was also significantly impaired in the severe hypoxia groups (Fig. 4B). With oral feedings of milk, the deficits ranged from some nasal regurgitation in the more mildly affected animals to an inability to retain fluid in the mouth in the more severely affected animals. We also observed that during feeding, the introduction of a plastic pipette into the side of the mouth of the control animal elicited an initial response in which the animal turned its head away from the pipette. Interestingly, this response was the most sensitive deficit that we observed, and it was significantly impaired in both the 30 $\min$ and $\geq 37$ min hypoxia groups (Fig. $4 C$ ).

\section{Evaluation of the general health of the P1 survivors of preterm uterine ischemia}

We conducted several studies to determine the effect of prior uterine ischemia on the general health of the P1 animals. The effect of uterine ischemia on intrauterine growth was investigated by comparing the weights of the survivors between the most severely affected group, $\geq 37 \mathrm{~min}$ at E22, with controls. There was a $6 \%$ difference in the weight of the live pups in the hypoxia group compared with controls that was related to the fact that the mean weight of the hypertonic pups was $14 \%$ less than the nonhypertonic pups in the hypoxia group. We then determined whether there were physiological differences between the control and ex- 

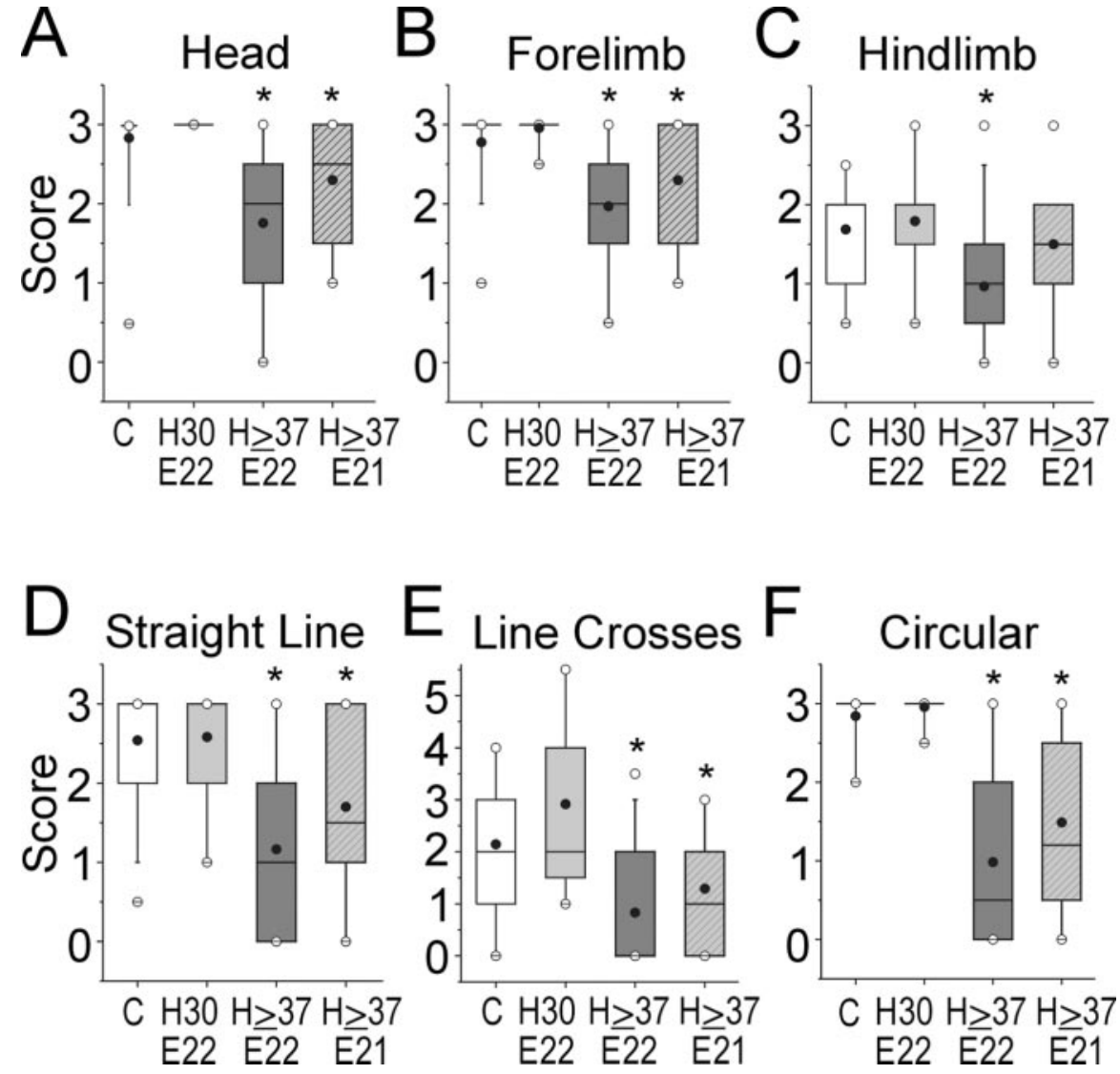

Figure 3. Motor activity score was assessed in a blinded manner in control pups (abscissa C) and after sustained hypoxia for 30 min at E22 ( $H 30 E 22), 37-40$ min at E22 ( $\mathrm{H} \geq 37 \mathrm{E22}$ ), or at E21 ( $\geq 37 \mathrm{E21}$ ). Score was based on assessment of the quality, intensity, and duration of head $(A)$, forelimb $(B)$, and hindlimb ( $C$ movements (see Table 3 for scoring). D shows the ability of a pup to move in a straight line. E shows the number of perpendicular line crosses in $60 \mathrm{sec}$ when walking forward in a straight line (see Table 3). F shows the ability to move in circles. Data are shown as a box and whisker plot. Black circles, Mean; white circles, 99th percentile. Groups are significantly different by Kruskal-Wallis test. ${ }^{*} p<0.0224$; Wilcoxon signed rank test compared with controls.

perimental groups that might affect performance during the behavioral testing. As summarized in Table 2, there were no significant differences between groups in the litter size or weight of the stillborn pups. In the animals tested, the core body temperature, heart rate, and mixed venous blood indices of $\mathrm{pH}, \mathrm{PcO}_{2}, \mathrm{Po}_{2}$, base excess, sodium, potassium, ionized calcium, and glucose were not different between the two groups.

\section{Sustained hypoxia at preterm gestation results in delayed cerebral injury at birth}

We next determined whether there was a pattern of delayed CNS injury that could account for the motor deficits sustained by the animals that survived for $10 \mathrm{~d}$ after a hypoxic insult of $40 \mathrm{~min}$ duration at E22. We surveyed four different levels of the P1 cerebral hemispheres and brain stem in five control animals and nine hypoxic animals (see Materials and Methods). An initial masked analysis of hematoxylin and eosin-stained paraffin embedded sections from these 14 animals showed little distinction between the control and hypoxic groups with the exception of distinct populations of activated microglia. Visualization of activated microglia and phagocytic macrophages by immunohistochemistry with a HAM 56 antibody confirmed the presence of distinct collections of these cells in the corona radiata, caudate putamen, ventral thalamic nuclei, hippocampal formation (dentate gyrus,
CA1, CA3, parasubiculum, and amygdalohippocampal area), and cerebellar hemispheres and vermis.

Figure 5 shows results from a blinded quantitative analysis of the number of HAM 56-labeled cells in the 14 animals (see Materials and Methods). On both sides of three brain regions from the hypoxic animals, the caudate putamen (Fig. $5 A$ ), ventral thalamic nuclei (Fig. $5 C, E, F$ ), and amygdalo-hippocampal area-parasubiculum (data not shown), there were similar significant elevations in the number of HAM 56-labeled cells. We also quantified the total area of HAM 56-labeled cells, because we reasoned that the area of the somata of activated microglia could be greater than that of resting microglia and, thus, could provide an additional means to identify regions of delayed injury. The results of this analysis demonstrated a similar significant elevation in the total area of HAM 56-labeled cells in the above three regions of the experimental group relative to control (data not shown). We did not observe collections of activated microglial in the subiculum, cranial nerve nuclei, ventral pons, inferior olivary nuclei, or anterior horn cells of the cervical cord.

Because this blinded histopathological analysis supported the fact that the basal ganglia and thalamus sustained delayed injury, we further evaluated the extent of injury to these regions with an additional marker of resting and activated microglia, isolectin B4. Numerous isolectin B4labeled cells were visualized in the head of the caudate nucleus, putamen, internal capsule, and in the anterior nuclei of the thalamus (data not shown). These cells were much more numerous and had a reactive hypertrophic appearance in the animals from the hypoxic group in contrast to the control animals in which the labeled cells had the appearance of resting microglia.

\section{Hypertonia at P1 correlates with injury to motor pathways}

We next asked whether the increase in the number of HAM 56-labeled cells in the caudate putamen and ventral thalamus of the hypoxia group at P1 was related to the motor disturbances seen in our model. We assigned thresholds of the 99th percentile of control cell number and tone of $\geq 2.5$ as the top limits of normality and did a $2 \times 2$ comparison of the number of HAM 56-labeled cells in the regions with tone. Forelimb hypertonia was highly correlated with an abnormal ( $>99$ th percentile of control cell number) increase in the number of HAM 56-labeled cells in both the caudate putamen (Fig. 5B) and thalamus (Fig. 5D). Similarly, hindlimb hypertonia was also highly correlated with an abnormal number of HAM 56labeled cells (data not shown). Interestingly, there was no correlation between an abnormal number of HAM 56-labeled cells in the amygdalo-hippocampal area-parasubiculum and hypertonia in either forelimbs or hindlimbs (data not shown). A similar analysis using 99th percentile of total area of control 

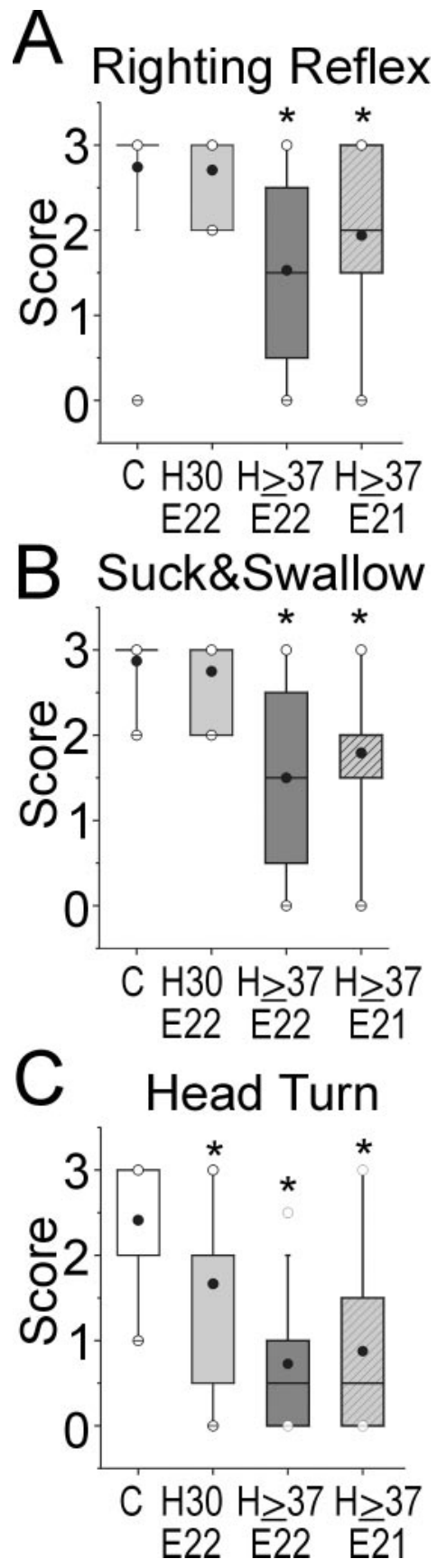

Figure 4. A, Righting reflex score assessing the ability of the pups to right themselves after being placed supine in control (abscissa C) and after sustained hypoxia for 30 min at E22 (H3O E22), 37- 40 min at E22 $\mathrm{d}(\mathrm{H} \geq 37 \mathrm{E22}$ ), or at E21 ( $\mathrm{H} \geq 37 \mathrm{E} 21)$. Similarly, $B$ shows the ability to suck and swallow. C shows the ability to reflexively turn the head away in response to introduction of a feeding dropper into the mouth. Data are shown as a box and whisker plot. Black circles, Mean; white circles, 99 th percentile. Groups are significantly different by KruskalWallis test. ${ }^{*} p<0.0224$; Wilcoxon signed rank test compared with controls.

versus hypertonia also showed similar results (data not shown).

Hypoxia-ischemia at E22 generates distinct patterns of acute injury to subcortical motor pathways

Because the forebrain of the neonatal survivors showed evidence of injury to motor pathways, we next determined whether these regions sustained acute injury in E22 fetal rabbits subjected to 40 min of hypoxia-ischemia. To visualize the magnitude and distri- bution of acute cell degeneration, TUNEL-labeled nuclei were visualized in animals that survived for $24 \mathrm{hr}$ (see Materials and Methods). Figure 6 summarizes schematically the typical distribution of TUNEL-labeled nuclei at two levels of the forebrain in these E23 fetal rabbits. The ventral forebrain consistently had a greater propensity for injury than did dorsal forebrain regions. Dorsal cortical structures typically had discrete focal lesions, whereas injury to ventral cortical structures, such as the piriform cortex, was more diffuse. In general, injury was primarily localized to cortical and subcortical gray matter structures. In contrast to animals that sustained hypoxia-ischemia, control animals had rare TUNEL-labeled cells in the regions shown in Figure 6. This finding was consistent with very similar data from the ovine fetal brain (Falkowski et al., 2002), which supports the concept that programmed cell death is quite low at this window in development.

Figure $7 A$ shows the typical distribution of TUNEL-labeled nuclei in the cortical plate. Degenerating nuclei were primarily localized to multifocal cortical lesions in the deep cortical mantle and the adjacent cortical subplate. Small periventricular lesions localized to the cortical neuroepithelium and the subventricular zone but did not involve the periventricular white matter. In the ventral forebrain, at the level of the septal nuclei (Fig. 6A), injury localized primarily to the piriform cortex, olfactory tubercle, caudate nucleus, caudate neuroepithelium (Fig. $7 B$ ), and ventral putamen (Fig. $7 C$ ). In the forebrain, at the level of the hipppocampus (Fig. $6 \mathrm{~B}$ ), pronounced subcortical injury localized to the caudate nucleus, caudate neuroepithelium, putamen, globus pallidus (Fig. 7D), and anterior thalamic nuclear group (Fig. 7E). Some animals also had small discrete hippocampal lesions that involved the CA1 and CA3 pyramidal cell layer (Fig. $7 F$ ) or the dentate gyrus. Less pronounced injury was also uncommonly observed in the anterior hypothalamic area and in the ventral medial nucleus of the hypothalamus (data not shown).

In summary, hypoxic-ischemia injury to the E22 fetus was not diffuse, but rather distinct regions of the fetal forebrain were consistently more vulnerable. Pronounced injury was most often observed in the basal ganglia and anterior thalamus with a lesser degree of injury in the cerebral cortex and hippocampus. Hence, injury to subcortical motor pathways was most commonly observed.

\section{Discussion}

This study demonstrates that in utero hypoxia-ischemia to the preterm rabbit fetus results in motor abnormalities in the newborn survivors. To our knowledge, this is the first model of antenatal hypoxic-ischemic injury to the developing brain that results in motor phenotypes, consistent with some of the motor impairments observed in children with CP. Most notably, hypertonia was observed in the survivors after hypoxia-ischemia at $67-70 \%$ gestation. This is consistent with human data in which preterm infants with a birth weight $<1500$ gm are $\sim 100$ times more likely to have CP than term infants of 3000-3500 gm (Cummins et al., 1993). In our model, impairment in locomotion, reflex motor activity, and coordination of suck and swallow were observed after hypoxia-ischemia in utero, similar to the clinical findings of hypoxic-ischemic encephalopathy in infants.

\section{Advantages of the newborn rabbit model}

The perinatal rabbit offers several distinct advantages relative to other animal models of hypoxic-ischemic brain injury. Rabbits and humans are perinatal brain developers, unlike rodents (postnatal) and sheep (prenatal) (van Marthens et al., 1975). Most importantly, newborn rabbits display a large repertoire of spontaneous motor activities and reflex responses that are reproduc- 

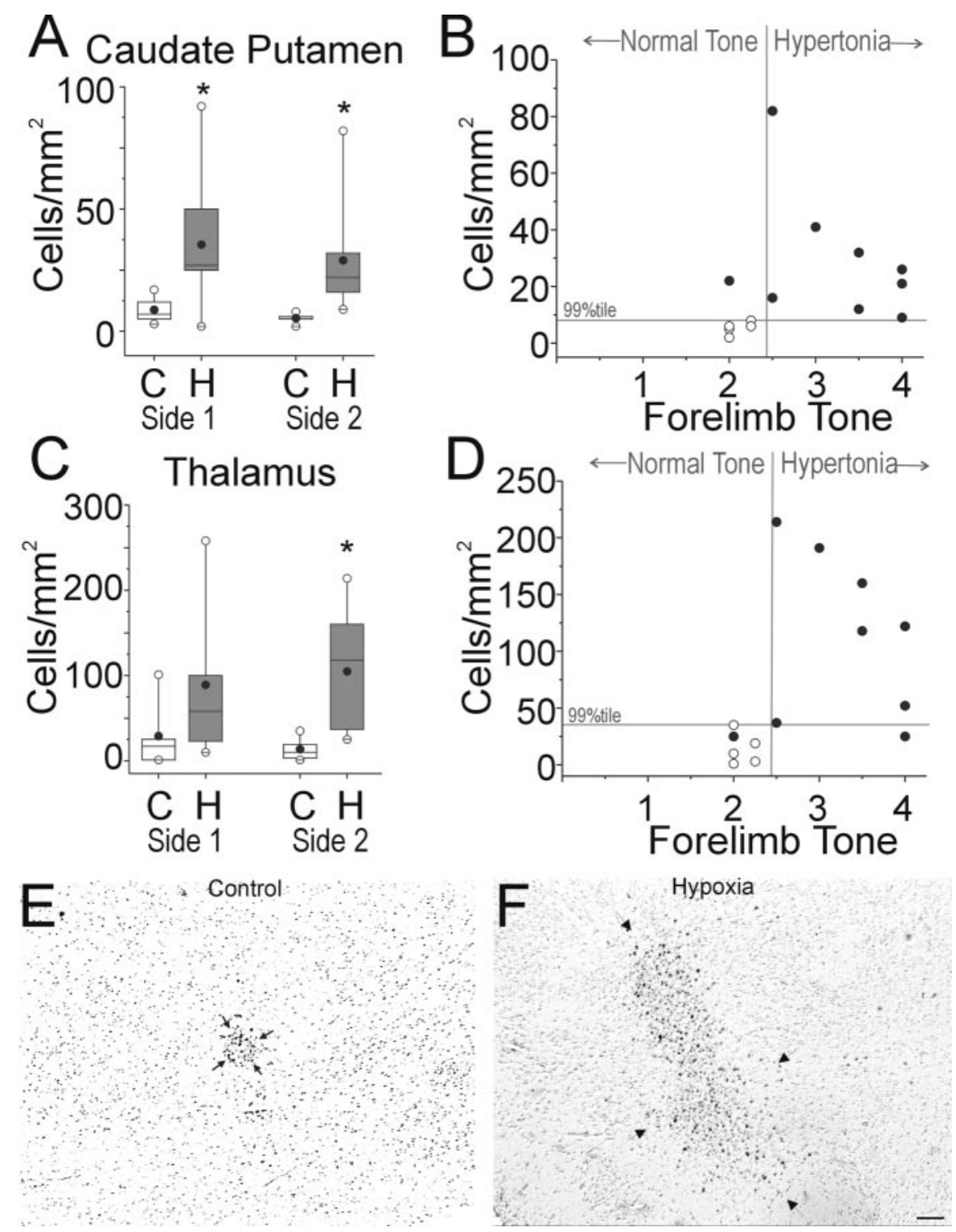

Figure 5. Blinded histopathological analysis reveals delayed injury to the caudate putamen and thalamus. Brain tissue sections obtained at P1 from control (C) and hypoxic survivors of 40 min hypoxia at E22 (H) were stained for HAM 56 antibody. Automated cell counts of HAM 56-labeled cells are shown as cell density (cells $/ \mathrm{mm}^{2}$ ) and were done as described in Materials and Methods. Side 1 and Side 2 refer to the counts obtained from each half of the brain, where the side was randomly assigned. $A$, Density of HAM 56-labeled cells in the caudate putamen. Data are shown as a box and whisker plot. Black circles, Mean; white circles, 99th percentile. ${ }^{*} p<0.05$; Wilcoxon signed rank test compared with controls. $B$, The association between the number of activated microglia and hypertonia is shown for the caudate putamen. Abscissa is tone with cutoff at 2.5 for defining hypertonia. Any point to the right of the vertical gray line represents a case of hypertonia. Ordinate represents cell density. A cutoff at the 99 th percentile for the control population is depicted as a horizontal gray line, and any point above this line represents a cell density that is higher than the 99th percentile of controls. The $2 \times 2$ association tested with Fisher's exact test is significant $(p<0.05)$. C, The density of HAM 56-labeled cells in the thalamus. Data are shown as a box and whisker plot. Black circles, Mean; white circles, 99th percentile. ${ }^{*} p<0.05$; Wilcoxon signed rank test compared with controls. $D$ is similar to $B$ and shows the association between the number of activated microglia in the ventral thalamus and hypertonia as described in the text. The abscissa and ordinate are as in $B$. The $2 \times 2$ association tested with Fisher's exact test is significant $(p<0.05)$. E and $F$ show representative images from the ventral thalamic nuclei of animals from the control and hypoxia groups, respectively. Arrows delineate the collections of HAM 56-labeled cells. Scale bars, $E, F, 100 \mu \mathrm{m}$.

ibly tested. Also, distinctive to newborn rabbits is the increased strength of the forelimbs compared with the hindlimbs. Perinatal locomotion progresses from an ability to move in circles, to moving in a straight line, to being able to jump, as presumably the hindlimbs become stronger. Jumping ability was significantly decreased in the hypoxic groups, and almost none of the animals from the $\geq 37$ min hypoxia group exhibited jumping behavior (data not shown).

\section{Timing of injury and}

maturation-dependent factors

The developmental maturation of motor systems in rabbits is accelerated relative to human. For example, gross motor control develops more rapidly in rabbits than in humans, and, by P3, coordinated movement of hindlimbs with forelimbs occurs. This is likely to contribute to the early appearance of motor abnormalities in rabbit survivors of hypoxia-ischemia.

Our neuropathological data are consistent with the emerging concept that the susceptibility of the developing brain to injury is maturation-dependent and involves preferential injury to, or sparing of, particular cell populations. Within the cerebral cortex, we observed a predilection of apparent subplate neurons to become TUNEL positive, consistent with recent observations that hypoxia-ischemia preferentially targets these neurons in the perinatal rat (McQuillen et al., 2003). Subplate neurons are critical for normal cortical development and have been implicated in the pathogenesis of $\mathrm{CP}$ related to human preterm cerebral injury (Allendoerfer and Shatz, 1994; Volpe, 1996). In humans, the high-risk period for cerebral white-matter injury coincides with the developmental window when late oligodendrocyte progenitors predominate in the white matter (Back et al., 2001). Interestingly, we did not observe a predilection for TUNELpositive cells in the cerebral white matter at E23 after hypoxia-ischemia at E22. One explanation could be that late oligodendrocyte progenitors are not a major population in the rabbit white matter until E24-E26 (S.A.B. and S.T., unpublished observations). Alternatively, cell death in the white matter may occur from delayed apoptosis, as previously observed in gray matter (Northington et al., 2001a).

\section{Hypoxia-ischemia to the preterm fetus targets subcortical motor pathways} After hypoxia-ischemia at E22, fetal rabbits sustained consistent acute injury to the basal ganglia and anterior thalamus, which are major components of subcortical motor pathways. At birth, behavioral abnormalities in the preterm survivors also correlated with delayed elevations in activated microglia in the basal ganglia and thalamus. These regions are particularly susceptible to hypoxic-ischemic injury in both preterm and term infants (Schneider et al., 1975; Rutherford et al., 1994; Okumura et al., 1997). The thalamus and basal ganglia are particularly susceptible to hypoxia-ischemic injury, as observed in postnatal rats with carotid artery ligation and hyp- 


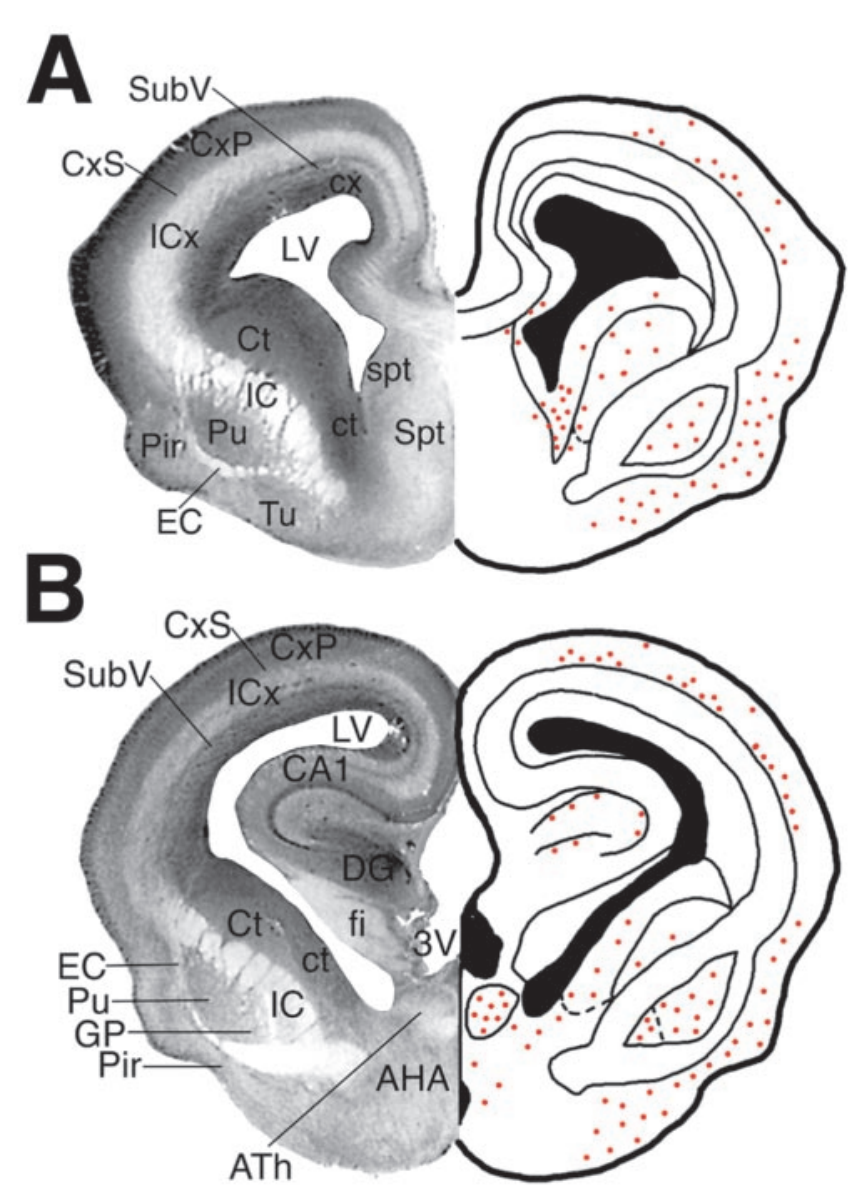

Figure 6. $A, B$, Nissl sections from the $\mathrm{E} 23$ rabbit fetus (left) and corresponding plots (right) of the distribution of TUNEL-labeled nuclei (red dots) in coronal sections of the forebrain at the level of the septal nuclei $(A)$ and at the level of the hippocampus $(B)$. Abbreviations were derived primarily from the classification scheme by Paxinos et al. (1994). AHA, Anterior hypothalamic area; ATh, anterior nuclear group of the thalamus; CA1, CA1 field of the hippocampus; CXP, cortical plate; CXS, cortical subplate; $C t$, caudate nucleus; ct, caudate neuroepithelium; DG, dentate gyrus; EC, external capsule; fi, fimbria of the fornix; GP, globus pallidus; IC, internal capsule; ICX, intermediate cortical zone; LV, lateral ventricle; Pir, piriform cortex; Pu, putamen; Spt, septum; spt, septal neuroepithelium; SubV, subventricular zone; $3 \mathrm{~V}$, third ventricle.

oxia (Northington et al., 2001b), in term fetal monkeys (Myers, 1975) and in preterm and near-term fetal sheep subjected to global cerebral hypoperfusion (Reddy et al., 1998).

\section{Nature of the hypertonic motor deficits}

Rabbit survivors of hypoxia-ischemia typically displayed hypertonia, which was elicited in both flexion and extension. The observed hypertonic deficits were consistent with the pronounced localization of injury to the basal ganglia and thalamus (for review see, Volpe, 2001). Our study was not designed to discriminate between subtypes of hypertonia, given the absence of universally accepted diagnostic protocols in children (Sanger et al., 2003). At rest, the majority of animals displayed increased flexor tone and flexion postures that were clinically reminiscent of the hypertonic contractures associated with spasticity. However, some animals displayed hypertonia without pronounced velocity-dependent resistance to limb flexion or extension, a feature of spastic hypertonia. The resistance to externally imposed joint movement was similar at low and high velocity in some animals, a feature of dystonic hypertonia (Sanger et al., 2003). In children, mixed hypertonia is more common than pure spastic hypertonia or pure dystonia (Sanger et al., 2003).

In human cerebral palsy, development of hypertonia and contractures develops months to years after a perinatal insult (Back, 1999). Because the hypoxic pups were unable to feed and survive after birth, it was not feasible to follow the evolution of motor abnormalities and neuropathology. This may be feasible in future studies with development of long-term intensive care support requiring ventilation (Bernard et al., 2003) and parenteral nutrition (Loff et al., 1998). In perinatal rodents that sustained hypoxicischemic cerebral injury, neuronal degeneration peaks within the first 24-72 hr in cortex, basal ganglia, and thalamus (Northington et al., 2001a; Han et al., 2002; McQuillen et al., 2003).

\section{Hypoxia-ischemia to the preterm fetus disrupts complex motor reflexes}

Newborn survivors of hypoxia-ischemia displayed significant disturbances in the coordination of suck and swallow. Many pups also displayed abnormal responses to oral motor stimulation. Our neuropathological studies support that oromotor dysfunction was related to supranuclear injury, because we failed to observe either acute or delayed injury to brainstem nuclei by TUNEL staining (data not shown). A more detailed study is needed to investigate the basis of such complex behavior that may be the summative response of many reflexes (Drewett et al., 1982; Keil et al., 1990; Kindermann et al., 1994). We also observed deficits in aversive head turning in response to noxious olfactory stimuli, such as peppermint or alcohol (data not shown), in the P1 survivors of hypoxia-ischemia. Future studies are needed to address whether the disruption of olfactory responses involved disturbances in motor integration, sensory integration, or both. Interestingly, $24 \mathrm{hr}$ after hypoxia-ischemia at E22, we observed extensive acute cell degeneration in the piriform cortex, which is the major region that integrates and transmits olfactory information from the olfactory bulb (Wilson, 2001)

\section{Behavioral deficits in other models of perinatal hypoxia-ischemia}

Previous studies have not generated an animal model that reproduces the motor abnormalities characteristic of CP. Perinatal rodents, neonatal piglets, and near-term fetal sheep and monkeys are highly susceptible to hypoxia-ischemia; however, a distinct phenotype of CP has not been defined (Raju, 1992; Back, 2001).

However, delayed behavioral abnormalities have been observed in many models (Nyakas et al., 1996). Antenatal hypoxia in $77 \%$ gestation rats resulted in increased locomotor activity at P13-P15, deficits in acquisition of passive avoidance, and increased swimming escape latency at P28 (Cai et al., 1999). Interestingly, antenatal hypoxia in rats does not result in histopathological abnormalities (Cai et al., 1999).

Postnatal rats readily sustain large hypoxic-ischemic histopathological lesions but do not exhibit hypertonia (Liu et al., 2001). Hypoxia-ischemia at P7 in rats results in cognitive deficits in short-term retention and swimming escape latency after 6 weeks (Liu et al., 2001). Intermittent hypoxia between 10-30 d resulted in similar spatial learning deficits on Morris water maze testing at $30 \mathrm{~d}$ (Row et al., 2002). Hypoxia-ischemia in 2-d-old rats resulted in motor deficits on rod, beam, and stair testing at 3 months (McQuillen et al., 2003).

\section{Stillbirths and hypoxia-ischemia}

In acute placental insufficiency such as abruptio placenta, pregnant women are 11 times more prone to stillbirths (Saftlas et al., 

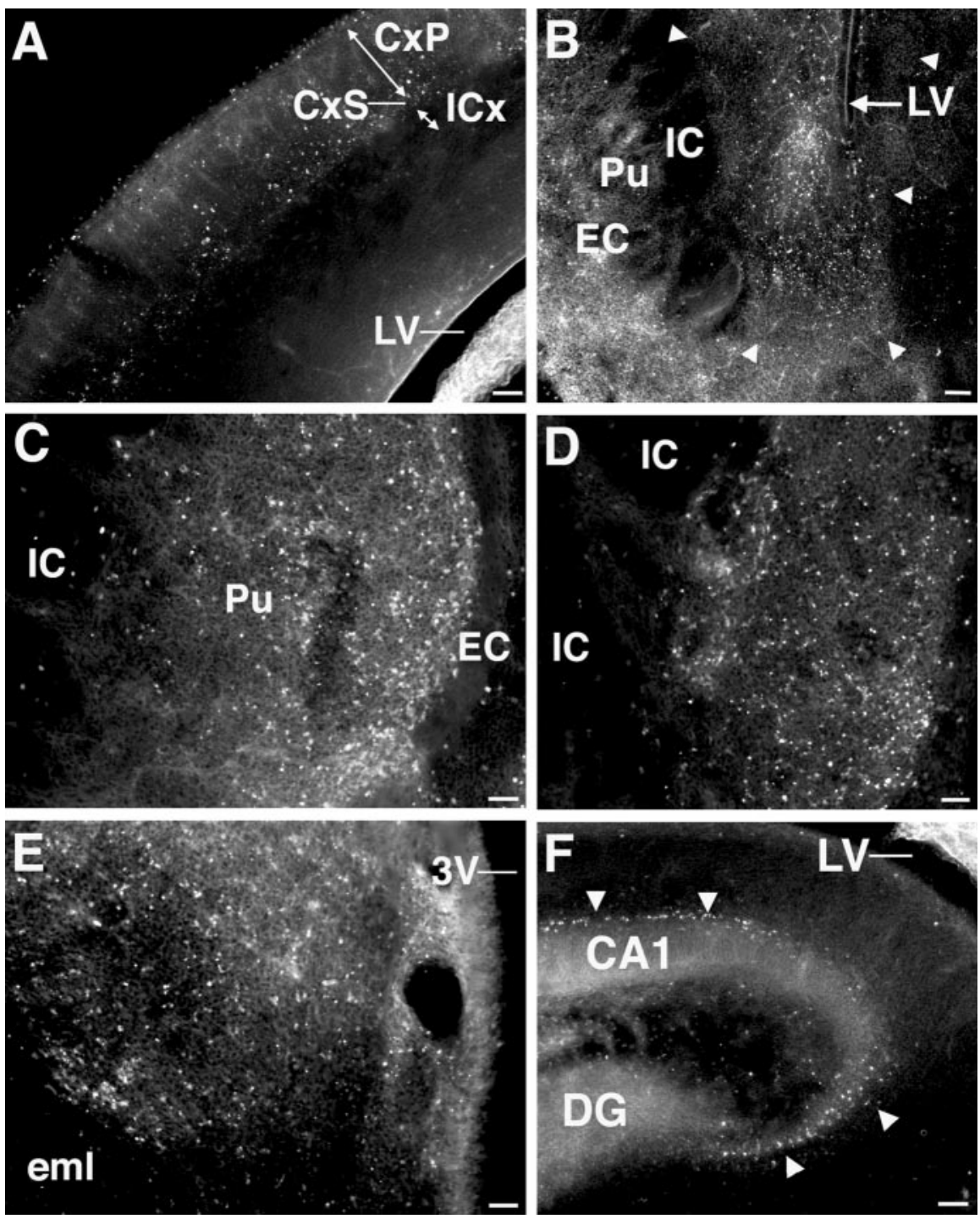

Figure 7. Fluorescent photomicrographs show the distribution of TUNEL-labeled nuclei in major structures of the E23 rabbit fetus that were injured after hypoxia-ischemia $24 \mathrm{hr}$ previously at E22. TUNEL labeling was negligible in the regions shown from E23 control fetuses (data not shown). A, In the cortical mantle, TUNEL labeling was concentrated deep in the cortical plate (CXP) and the adjacent subplate layer (CXS) in discrete multifocal lesions. Note that the intermediate cortical layer (ICX) that will give rise to the corona radiata is spared. LV, Lateral ventricle. $B$, TUNEL staining in the ventral caudate neuroepithelium (demarcated by arrowheads) situated between the internal capsule (IC) and the lateral ventricle (LV). Increased TUNEL staining is also seen lateral to the external capsule (bottom left) in the piriform cortex. C, TUNEL staining in the putumen (Pu). Note that the white matter tracts of the external capsule (EC) and internal capsule (IC) are mostly spared. D, TUNEL staining was increased in the globus pallidus; however, the adjacent white matter tracts of the internal capsule (IC) were unlabeled. E, Extensive TUNEL staining in the anterior nuclear group of the thalamus. eml, External medullary lamina; 3V, third ventricle. F, Scattered TUNEL-labeled nucle were visualized in the hippocampus in the pyramidal cell layer in the CA1 and CA3 fields (arrowheads). DG, Dentate gyrus. Scale bars: $A, B, F, 100 \mu \mathrm{m} ; C-E, 50 \mu \mathrm{m}$.

1991). Perinatal anoxia was a major cause of stillbirths and early neonatal deaths (Gunn and Cable, 1984). The present study confirms the correlation between the time of insult and the stillbirth rate (Fig. $1 A$ ). Thus, the extent of neurobehavioral abnormalities reported in our study may actually be underestimated, because the most affected animals probably died. Consistent with this possibility, some of our stillbirths had tonic postures similar to the survivors (not contractures, because the limb could be passively extended). Vigorous resuscitation of unexpected stillbirths in humans has shown that the majority of survivors have longterm disability (Casalaz et al., 1998).

\section{Clinical relevance}

In summary, we generated a model of antenatal hypoxia at preterm gestation that resulted in perinatal deaths and a phenotype in the survivors that resembles the early manifestations of CP. This model is consistent with the emerging clinical concept that antenatal factors are pathogenetically related to the subsequent development of CP in many patients. Thus, our model provides an important behavioral endpoint that has heretofore been inaccessible to study. Future studies are needed to determine the cellular and molecular mechanisms that precede $\mathrm{CP}$ and to assess whether these deficits can be ameliorated by therapeutic interventions.

\section{References}

Allendoerfer KL, Shatz CJ (1994) The subplate, a transient neocortical structure: its role in the development of connections between thalamus and cortex. Annu Rev Neurosci 17:185-218.

Back SA (1999) Cerebral palsy. In: Developmental-behavioral pediatrics (Levine $\mathrm{MD}$, Carey WB, Crocker AC, eds), pp 579-588. Philadelphia: Saunders.

Back SA (2001) Recent advances in human perinatal white matter injury. Prog Brain Res 132:131-147.

Back SA, Luo NL, Borenstein NS, Levine JM, Volpe JJ, Kinney HC (2001) Late oligodendrocyte progenitors coincide with the developmental window of vulnerability for human perinatal white matter injury. J Neurosci 21:1302-1312.

Badawi N, Kurinczuk JJ, Keogh JM, Alessandri LM, O'Sullivan F, Burton PR, Pemberton PJ, Stanley FJ (1998) Antepartum risk factors for newborn encephalopathy: the Western Australian case-control study. $\mathrm{Br}$ Med J 317:1549-1553.

Bernard N, Matecki S, Py G, Lopez S, Mercier J, Capdevila X (2003) Effects of prolonged mechanical ventilation on respiratory muscle ultrastructure and mitochondrial respiration in rabbits. Intensive Care Med 29:111-118.

Cai Z, Xiao F, Lee B, Paul IA, Rhodes PG (1999) Prenatal hypoxia-ischemia alters expression and activity of nitric oxide synthase in the young rat brain and causes learning deficits. Brain Res Bull 49:359-365.

Casalaz DM, Marlow N, Speidel BD (1998) Outcome of resuscitation following unexpected apparent stillbirth. Arch Dis Child Fetal Neonatal Ed 78:F112-F115.

Cummins SK, Nelson KB, Grether JK, Velie EM (1993) Cerebral palsy in four northern California counties, births 1983 through 1985. J Pediatr 123:230-237.

Damiano DL, Quinlivan JM, Owen BF, Payne P, Nelson KC, Abel MF (2002) What does the Ashworth scale really measure and are instrumented measures more valid and precise? Dev Med Child Neurol 44:112-118.

Derrick M, He J, Brady E, Tan S (2001) The in vitro fate of rabbit fetal brain cells after acute in vivo hypoxia. J Neurosci 21:1-5.

Drewett RF, Kendrick KM, Sanders DJ, Trew AM (1982) A quantitative analysis of the feeding behavior of suckling rabbits. Dev Psychobiol 15:25-32

Falkowski A, Hammond R, Han V, Richardson B (2002) Apoptosis in the preterm and near term ovine fetal brain and the effect of intermittent umbilical cord occlusion. Dev Brain Res 136:165-173. 
Gunn T, Cable G (1984) Perinatal mortality at a level 2 obstetric hospital: problems after 32 weeks gestation. N Z Med J 97:862-865.

Han BH, Xu D, Choi J, Han Y, Xanthoudakis S, Roy S, Tam J, Vaillancourt J, Colucci J, Siman R, Giroux A, Robertson GS, Zamboni R, Nicholson DW, Holtzman DM (2002) Selective, reversible caspase-3 inhibitor is neuroprotective and reveals distinct pathways of cell death after neonatal hypoxic-ischemic brain injury. J Biol Chem 277:30128-30136.

Keil W, von S, Hudson R (1990) A behavioral bioassay for analysis of rabbit nipple-search pheromone. Physiol Behav 47:525-529.

Kindermann U, Hudson R, Distel H (1994) Learning of suckling odors by newborn rabbits declines with age and suckling experience. Dev Psychobiol 27:111-122.

Liu XH, Eun BL, Barks JD (2001) Platelet-activating factor antagonist bn 50730 attenuates hypoxic-ischemic brain injury in neonatal rats. Pediatr Res 49:804-811.

Loff S, Waag KL, Kranzlin B, Zovko D, Dzakovic A, Jester I, Wirth H, Wessel L (1998) Long-term total parenteral nutrition-induced hepatobiliary dysfunction in a rabbit model. J Pediatr Surg 33:694-699.

McQuillen PS, Sheldon RA, Shatz CJ, Ferriero DM (2003) Selective vulnerability of subplate neurons after early neonatal hypoxia-ischemia. J Neurosci 23:3308-3315.

Myers RE (1975) Fetal asphyxia due to umbilical cord compression. Metabolic and brain pathologic consequences. Biol Neonate 26:21-43.

Northington FJ, Ferriero DM, Graham EM, Traystman RJ, Martin LJ (2001a) Early neurodegeneration after hypoxia-ischemia in neonatal rat is necrosis while delayed neuronal death is apoptosis. Neurobiol Dis 8:207-219.

Northington FJ, Ferriero DM, Martin LJ (2001b) Neurodegeneration in the thalamus following neonatal hypoxia-ischemia is programmed cell death. Dev Neurosci 23:186-191.

Nyakas C, Buwalda B, Luiten PG (1996) Hypoxia and brain development. Prog Neurobiol 49:1-51.

Okumura A, Hayakawa F, Kato T, Kuno K, Watanabe K (1997) MRI findings in patients with spastic cerebral palsy. I. Correlation with gestational age at birth. Dev Med Child Neurol 39:363-368.

Paxinos G, Ashwell KWS, Tork I (1994) Atlas of the developing rat nervous system. San Diego: Academic.

Raju TN (1992) Some animal models for the study of perinatal asphyxia. Biol Neonate 62:202-214.

Reddy K, Mallard C, Guan J, Marks K, Bennet L, Gunning M, Gunn A, Gluckman P, Williams C (1998) Maturational change in the cortical response to hypoperfusion injury in the fetal sheep. Pediatr Res 43:674-682.
Robertson C, Finer N (1985) Term infants with hypoxic-ischemic encephalopathy: outcome at 3.5 years. Dev Med Child Neurol 27:473-484.

Row BW, Kheirandish L, Neville JJ, Gozal D (2002) Impaired spatial learning and hyperactivity in developing rats exposed to intermittent hypoxia. Pediatr Res 52:449-453.

Rutherford MA, Pennock JM, Dubowitz LM (1994) Cranial ultrasound and magnetic resonance imaging in hypoxic-ischaemic encephalopathy: a comparison with outcome. Dev Med Child Neurol 36:813-825.

Saftlas AF, Olson DR, Atrash HK, Rochat R, Rowley D (1991) National trends in the incidence of abruptio placentae, 1979-1987. Obstet Gynecol 78:1081-1086.

Sanger TD, Delgado MR, Gaebler-Spira D, Hallett M, Mink JW, Task F (2003) Classification and definition of disorders causing hypertonia in childhood. Pediatrics 111:e89-e97.

Schmidt B, Davis P, Moddemann D, Ohlsson A, Roberts RS, Saigal S, Solimano A, Vincer M, Wright LL (2001) Long-term effects of indomethacin prophylaxis in extremely-low-birth-weight infants. N Engl J Med 344:1966-1972.

Schneider H, Ballowitz L, Schachinger H, Hanefeld F, Droszus JU (1975) Anoxic encephalopathy with predominant involvement of basal ganglia, brain stem and spinal cord in the perinatal period. Report on seven newborns. Acta Neuropathologica 32:287-298.

Tan S, Zhou F, Nielsen VG, Wang Z, Gladson CL, Parks DA (1998) Sustained hypoxia-ischemia results in reactive nitrogen and oxygen species production and injury in the premature fetal rabbit brain. J Neuropathol Exp Neurol 57:544-553.

Tan S, Zhou F, Nielsen VG, Wang Z, Gladson CL, Parks DA (1999) Increased injury following intermittent fetal hypoxia-reoxygenation is associated with increased free radical production in fetal rabbit brain. J Neuropathol Exp Neurol 58:972-981.

Tan S, Bose R, Derrick M (2001) Hypoxia-ischemia in fetal rabbit brain increases reactive nitrogen species production: quantitative estimation of nitrotyrosine. Free Radic Biol Med 30:1045-1051.

van Marthens E, Harel S, Zamenshof S (1975) Experimental intrauterine growth retardation. Biol Neonate 26:221-231.

Volpe JJ (1996) Subplate neurons-missing link in brain injury of the premature infant? Pediatrics 97:112-113.

Volpe JJ (2001) Neurobiology of periventricular leukomalacia in the premature infant. Pediatr Res 50:553-562.

Wilson DA (2001) Receptive fields in the rat piriform cortex. Chem Senses 26:577-584.

Wright J, Rang M (1990) The spastic mouse. And the search for an animal model of spasticity in human beings. Clin Orthop 253:12-19. 